\title{
Tumor Necrosis Factor- $\alpha$ and IL-17A Activation Induces Pericyte-Mediated Basement Membrane Remodeling in Human Neutrophilic Dermatoses
}

Holly M. Lauridsen, ${ }^{*}$ Amanda S. Pellowe, ${ }^{*}$ Anand Ramanathan, ${ }^{*}$ Rebecca Liu ${ }^{\dagger}$ Kathryn Miller-Jensen, ${ }^{*}$ Jennifer M. McNiff, Jordan S. Pober, ${ }^{\dagger *}$ and Anjelica L. Gonzalez*

From the Departments of Biomedical Engineering* and Immunobiology, ${ }^{\dagger}$ Yale University, New Haven; and the Department of Dermatology, ${ }^{\ddagger}$ Yale School of Medicine, New Haven, Connecticut

\author{
Accepted for publication \\ April 24, 2017. \\ Address correspondence to \\ Anjelica L. Gonzalez, Ph.D., \\ Donna L. Dubinsky Department \\ of Biomedical Engineering, \\ Yale University, Malone Engi- \\ neering Center, PO Box \\ 208260, New Haven, CT \\ 06520-8260. E-mail: anjelica. \\ gonzalez@yale.edu.
}

\begin{abstract}
Sweet syndrome (SS) is a prototypical neutrophilic dermatosis, a class of inflammatory diseases marked by elevated levels of tumor necrosis factor (TNF)- $\alpha$ and IL-17A, pathologic neutrophil recruitment, and microvascular remodeling. Histologic analyses of four matrix proteins-collagen I and IV, laminin, and fibronectin - in skin biopsies of patients with SS reveal that the basement membrane of dermal postcapillary venules undergoes changes in structure and composition. Increased neutrophil recruitment in vivo was associated with increases in collagen IV, decreases in laminin, and varied changes in fibronectin. In vitro studies using TNF- $\alpha$ and IL-17A were conducted to dissect basement membrane remodeling. Prolonged dual activation of cultured human pericytes with TNF- $\alpha$ and IL-17A augmented collagen IV production, similar to in vivo remodeling. Co-activation of pericytes with TNF- $\alpha$ and IL-17A also elevated fibronectin levels with little direct effect on laminin. However, the expression of fibronectin- and laminin-specific matrix metalloproteinases (MMPs), particularly MMP-3, was significantly upregulated. Interactions between pericytes and neutrophils in culture yielded even higher levels of active MMPs, facilitating fibronectin and laminin degradation, and likely contributing to the varied levels of detectable fibronectin and the decreases in laminin observed in vivo. These data indicate that pericyteneutrophil interactions play a role in mediating microvascular changes in SS and suggest that targeting MMP-3 may be effective in protecting vascular wall integrity. (Am J Pathol 2017, 187: 1893-1906; http://dx.doi.org/10.1016/j.ajpath.2017.04.008)
\end{abstract}

Neutrophilic dermatoses (NDs) describe diverse inflammatory disorders of the skin that commonly feature recruitment of neutrophils but lack the destruction of microvessels associated with leukocytoclastic vasculitis. ${ }^{1}$ Included within this family are Behçet disease, pyoderma gangrenosum, and Sweet syndrome (SS); SS is frequently considered a prototypical member of this family. ${ }^{2,3}$ The onset of SS is often idiopathic, but certain populations are more susceptible. Women, particularly pregnant women, are more prone to SS and represent $80 \%$ of patients with classic SS. Nearly onethird of patients with SS have a systemic inflammatory disease, such as Crohn disease or rheumatoid arthritis, suggesting a systemic elevation in immune response that presents as localized neutrophil specific inflammation. ${ }^{1,4}$ An additional $20 \%$ may have a hematologic malignant tumor, suggestive of a paraneoplastic syndrome. ${ }^{1}$ The initial presentation of SS is clinically marked with painful erythematous lesions across the body, notably on the hands and upper extremities. ${ }^{4-6}$ Histologically, these lesions exhibit excessive neutrophil accumulation into a perivascular space of the dermis. ${ }^{7,8}$ The lesions and perilesional regions are

Supported by NIH National Heart Lung and Blood Institute grant R01 HL051014 (J.S.P., R.L., and A.L.G.), NIH Medical Scientist Training Program grant T32-GM007205 (R.L.), NIH National Research Service Award predoctoral fellowship F31-HL129563 (R.L.), and the Donna L. Dubinsky Initiative (A.L.G.).

H.M.L. and A.S.P. contributed equally to this work.

Disclosures: None declared. 
rich in proinflammatory cytokines, specifically, tumor necrosis factor (TNF)- $\alpha$ and IL-17A., ${ }^{2,9,10}$

Although the absence of vascular damage has historically been used to distinguish SS from vasculitis, this criterion has been recently reevaluated. ${ }^{8}$ In the analysis of 21 patient biopsy specimens, Malone et $\mathrm{al}^{8}$ found that nearly $80 \%$ of patients studied had some evidence of vascular wall damage, including the presence of intramural neutrophils and/or extravasated red blood cells. These observations suggest that microvascular injury associated with SS is a secondary effect of leukocyte recruitment rather than a driver of lesion formation.

Postcapillary venules are the primary site of neutrophil recruitment in skin inflammation. ${ }^{11,12} \mathrm{PCV}$ s are composed of a luminal endothelial cell (EC) lining surrounded by an outer layer of pericytes (PCs) embedded within a shared basement membrane (BM). ${ }^{13,14}$ Recent studies have emphasized that the ability of neutrophils to successfully traverse the PCV wall involves interactions with ECs, PCs, and $\mathrm{BM}$, as well as indirect signaling among these components to define the overall inflammatory environment within the vascular wall and perivascular region. ${ }^{13,15,16}$ Neutrophil-EC interactions dominate the initial steps of leukocyte recruitment and have been the focus of many prior investigations of the leukocyte adhesion cascade. In brief, cytokine activation induces human ECs to rapidly synthesize and display E-selectin, which tethers circulating neutrophils. ${ }^{17}$ Cytokine activation of ECs also results in the up-regulation of leukocyte adhesion molecules that serve as integrin ligands (eg, intercellular adhesion molecule 1 and vascular cell adhesion molecule 1) and in augmented EC production of IL-8 that supports leukocyte activation and crawling along the EC lining. ${ }^{18}$ Junctional molecules between ECs, such as CD99 and platelet endothelial cell adhesion molecular 1, facilitate neutrophil transendothelial migration while maintaining the barrier function of the EC layer. ${ }^{19}$

The ensuing steps in neutrophil recruitment-migration across PCs and the BM-have been less well evaluated, although initial studies have confirmed that the roles of PC and $\mathrm{BM}$ are critical and active. ${ }^{13,15,20,21}$ Located at the interface of the vessel and the perivascular extracellular matrix (ECM), PCs are strategically positioned to affect leukocyte recruitment. Thus far, it has been demonstrated that cytokine-activated PCs produce IL- $8^{20,22}$; up-regulate leukocyte adhesion molecules, including intercellular adhesion molecule $1^{20,23}$; and provide guidance to migrating neutrophils. ${ }^{24}$ Furthermore, PCs synthesize, reorganize, and potentially degrade matrix proteins and therefore affect the biochemical and structural composition of the microvascular BM and the ability of neutrophils to migrate through it. ${ }^{25,26}$ In addition, it has been widely recognized that neutrophils are capable of remodeling their microenvironment through degranulation and release of primary, secondary, and tertiary granule contents, which can include MMPs. However, the combined effects of PCs and neutrophils on BM remodeling remain unexplored.

We recently reported that PCs, but not ECs, mount a robust paracrine response to IL-17A, and this response often synergizes with the response to TNF- $\alpha$. Among other changes, IL-17A-activated PCs increase their production of granulocyte colony-stimulating factor, granulocytemacrophage colony-stimulating factor, IL-6, and IL-8. ${ }^{27}$ Consequently, PC-paracrine signals are implicated in neutrophil chemotaxis, prolonging of neutrophil survival, and augmentation of neutrophil production of proinflammatory moieties, including TNF- $\alpha$ and IL-6. To date, the ability of TNF- $\alpha$ and IL-17A to induce remodeling of the BM has not been reported.

We use histologic analysis of human dermal microvasculature to quantify the degree of vascular remodeling in dermal PCVs present in SS. We analyze seven patient skin biopsy specimens for pathologic perturbations in collagen type I and IV, laminin, and fibronectin, as well as the presence of neutrophil specific myeloperoxidase (MPO). We then assess the dynamic, compositional, and structural remodeling ability of human PCs and neutrophils after temporal activation with TNF- $\alpha$ and IL-17A.

\section{Materials and Methods}

\section{Histologic Analysis}

Paraffin-embedded archival biopsy specimens from seven deidentified patients previously diagnosed with SS were obtained from the Department of Dermatology, Yale University. Control samples, also deidentified, were obtained from patients who were not diagnosed with SS, denoted as healthy volunteers in this article. The use of these materials is classified as not human subjects research by the Yale Human Investigations Committee. Paraffin-embedded sections were stained for human collagen type IV (Dako, Santa Clara, CA), laminin (AbCam, Cambridge, MA), fibronectin $(\mathrm{AbCam}), \alpha$-smooth muscle actin $(\alpha$-SMA; ThermoFisher, Waltham, MA), Masson's Trichrome and MMP-3 (AbCam), and MPO (ThermoFisher), and mounted. Samples were imaged at $\times 40$ magnification and stitched together using Image Composite Editor version 2.0 (Microsoft, Redmond, WA). Qualitative analyses of the vessel integrity at sections visible within the histologic slices were made using all four histologic stains. Qualitative assessment of the vessel integrity was denoted by a metric known as the remodeling index (RI), ranging from 1 to 3 . Vessels with an RI of 1 had appropriate vascular structure; minimal leukocyte recruitment in the periregional space was observed. Vessels scored with an RI of 2 had some degree of vascular remodeling or damage; the presence of thickened venule walls, loose fibrils, or cellular processes extending from the cell or intramural erythrocytes were used as indications of an RI equal to 2. Lastly, vessels scored with an RI of 3 indicated severe disruption of the vessel 
wall, disengagement of the pericyte via a loss of the circumferential connection to the endothelium and BM of the microvascular wall, and the presence of erythrocytes in the perivascular space. Analysis of staining intensity was completed with ImageJ software version $1.5(\mathrm{NIH}$, Bethesda, MD; http://imagej.nih.gov/ij) using the IHC Image Analysis Toolbox plug-in (https://imagej.nih.gov/ij/ plugins/ihc-toolbox/index.html), which isolates positively stained sections from the remainder of the tissue. ${ }^{28}$ In brief, regions of interest were created for individual PCVs in the superficial plexus. Approximate vessel locations in the corresponding MPO-stained sections were then identified, and the intensity of local MPO staining was measured. PCVs were identified by their localized positioning within the superficial plexus, the vascular morphologic features, microvascular diameter, and the presence of visible PCs surrounding a luminal EC. The age, sex, and location of the lesion were only made available after the analysis was complete. The patient sample size was determined before analysis, but the number of vessels analyzed was not.

\section{PC Isolation, Culture, and Identification}

Human placental PCs were isolated via microvessel explantoutgrowth methods previously described. ${ }^{29}$ In brief, fresh, deidentified human placentas were rinsed thoroughly with phosphate-buffered saline (PBS; Corning, Corning, NY) to remove red blood cells. Tissue was then finely minced and incubated in collagenase D (Roche, Indianapolis, IN) to digest the tissue. Subsequently, microvessels were isolated from the tissue mixture via sequential filtering and size exclusion techniques. Microvessels remained in culture for 2 weeks with PC complete media (M199; Gibco, Grand Island, NY) supplemented with $20 \%$ fetal bovine serum (Hyclone Laboratories, Inc., Logan, UT) and 1\% penicillinstreptomycin (Gibco). PCs were then used between passages 3 and 8. The isolation and use of these cells are classified as not human subjects research by the Yale Human Investigations Committee.

Isolated human PCs were phenotyped using flow cytometry. After culture, PCs were washed with PBS, trypsinized, and resuspended in $4 \%$ paraformaldehyde (PFA) for $30 \mathrm{mi}-$ nutes. Cells were centrifuged and incubated in $2 \%$ bovine serum albumin (BSA; Santa Cruz Biotechnology, Dallas, TX) for at least 1 hour before staining. Cells were resuspended in $2 \%$ BSA with anti-human $\alpha$-SMA (clone 1A4; Santa Cruz Biotechnology), CD90 (clone K-15; Santa Cruz Biotechnology), or neuron glial antigen (NG)-2 (clone H-300; Santa Cruz Biotechnology) overnight at $4^{\circ} \mathrm{C}$. Cells were subsequently incubated in 2\% BSA with anti-mouse fluorescein isothiocyanate (Sigma-Aldrich, St. Louis, MO), anti-goat Alexa Fluor 647 (Invitrogen, Grand Island, NY), or antirabbit Alexa Fluor 647 (BioLegend, San Diego, CA) for 1 hour at room temperature. All samples were resuspended in PBS and filtered, and at least 5000 cells were analyzed on an
S1000EX STD-13+L flow cytometer (Stratedigm, San Jose, CA). Positive fluorescence was normalized to IgG controls.

To confirm similarity between human placental PCs and human dermal PCs, deidentified adult human skin samples were acquired from the tissue procurement service of the Department of Pathology, Yale University. Skin was dermatomed to a thickness of $0.5 \mathrm{~mm}$. Fresh-frozen tissues were embedded in optimum cutting temperature compound (VWR International, Radnor, PA), and tissue sections were prepared $(5.0 \mu \mathrm{m})$. For immunofluorescence imaging, tissues were incubated with anti- $\alpha$-SMA (clone 1A4; Sigma-Aldrich), anti-Thy-1 (clone F15-42-1, Millipore, Mahopac, NY), NG2 antibody (clone 7.1; Santa Cruz Biotechnology), and fluorescein- or rhodamine-labeled ULEX (Vector Laboratories, Burlingame, CA) overnight at $4^{\circ} \mathrm{C}$. Appropriate secondary antibodies (Life Technologies, Carlsbad, CA) were used to detect primary antibodies, and glass coverslips were mounted for analysis in ProLong mounting media (Life Technologies).

To evaluate PC-deposited protein in vitro, PCs were cultured on 25-mm glass coverslips (VWR International) for protein quantification and $25-\mathrm{mm}$ polycarbonate transwells (Corning). Confluent culture flasks of PCs were trypsinized (0.25\% trypsin-EDTA; Gibco) and seeded on coverslips or transwells at a concentration of 300,000 cells $/ \mathrm{mL}$. Coverslips were cultured in six-well plates with agarose-coated wells to prevent PC overgrowth into the well; PCs on transwells were contained within the transwell apical chamber. PCs were fed with PC complete media supplemented with $100 \mu \mathrm{mol} / \mathrm{L}$ ascorbic acid every other day. PCs were cultured under nonactivated conditions for 8 days to establish a baseline layer of protein. After the establishment of this protein layer, PCs were cultured under nonactivating, TNF- $\alpha$-activating (10 ng/mL), IL-17A-activating ( $20 \mathrm{ng} / \mathrm{mL})$, or TNF- $\alpha$ plus IL-17A-activating (10 and $20 \mathrm{ng} / \mathrm{mL}$, respectively) conditions for 1,3 , or 5 days.

\section{PC Protein Assessment}

To assess PC protein deposition on glass coverslips, PCs were fixed in 4\% PFA, blocked with BSA, and stained with anti-human collagen type I, collagen type IV, laminin, and fibronectin antibodies. Samples were then stained with appropriate Li-COR secondary antibodies for a LI-COR Odyssey flatbed scanner (LI-COR, Lincoln, NE). Samples were scanned and protein analysis was quantified using LI-COR Image Studio Lite software version 5.2 (LI-COR).

Protein alignment was determined after PCs were fixed in 4\% PFA. To assess collagen type IV, laminin, and fibronectin alignment, coverslips were similarly blocked and stained with anti-human primary antibodies and appropriate fluorescein isothiocyanate or tetramethylrhodamine-tagged secondary antibodies. Coverslips were mounted onto glass slides with Vectashield soft mounting media (Vector Laboratories). Slides were imaged on a Zeiss Axiovert inverted 
fluorescent microscope using Axiovision software version 4.8. For collagen type I alignment, samples were fixed with 4\% PFA and stained using a Picosirius Red kit according to the manufacturer's instructions (Polysciences, Inc., Warrington, PA). PC protein samples were imaged under polarized (Picosirius Red) or fluorescent light, split into red, blue, and green channels using ImageJ software, and analyzed for isotropy via a fast Fourier transform in Matlab (Mathworks, Natick, MA) as previously described. ${ }^{30}$

To assess pore diameter, samples were fixed with Hollande's fixative and then stained with eosin to fluorescently tag all protein in the sample. Samples were then mounted and imaged on a Nikon Spinning Disk Confocal Microscope (Nikon, Tokyo, Japan). Pores were measured using ImageJ software. All samples have a minimum sample size of three.

\section{PC Proliferation}

Three methods were used to determine whether cytokine activation of PCs altered cell survival or proliferation. First, PCs were labeled with CellTrace carboxyfluorescein succinimidyl ester staining (Life Technologies). In brief, before seeding, PCs were labeled in $5 \mu \mathrm{mol} / \mathrm{L}$ carboxyfluorescein succinimidyl ester staining solution for 15 minutes at $37^{\circ} \mathrm{C}$. Cells were centrifuged to remove the staining solution, resuspended in media, and incubated for 5 minutes. Cells were once again centrifuged and seeded at confluence in six-well plates. PCs were activated for 5 days using the concentrations previously specified in PC Isolation, Culture, and Identification. PCs were then trypsinized and fixed. Carboxyfluorescein succinimidyl ester stain intensity (488-nm excitation) was measured on an LSRII flow cytometer with BD Diva software version 8.0.1 (BD Biosciences, Franklin Lakes, NJ). Second, PC samples prepared for LI-COR protein analysis were fixed and stained with DAPI to identify cell nuclei. Samples were imaged at random locations using a Zeiss Axiovert inverted fluorescent microscope equipped with Axiovision software. Nuclei were counted to determine the number of cells per sample. Third, the total protein content of the soluble cell lysate (described below) as determined by bicinchoninic acid (BCA) assay (Fisher Scientific, Waltham, $\mathrm{MA}$ ), was used to compare the relative cellular protein content of PC cultures.

\section{Neutrophil Isolation and Chemotaxis}

Fresh human neutrophils were isolated from the peripheral blood of healthy adult volunteers in accordance with a protocol approved by Yale's Institutional Review Board. Peripheral vein blood was drawn into syringes that contained citrate phosphate dextrose and 6\% dextran (Polysciences, Inc.). ${ }^{13}$ Syringes were incubated upright to allow the hematocrit to settle from the plasma. Plasma was subsequently collected and centrifuged. The remaining pellet was resuspended in PBS and layered on a Histopaque 1077 column (Sigma-Aldrich) and centrifuged. Once again, the neutrophil-containing pellet was collected and resuspended in PBS and centrifuged once again. The remaining cell population was resuspended in PBS supplemented with magnesium, calcium, and glucose at a final concentration of 6 million cells per millilieter.

PC protein was grown on transwells according to the activation strategies outlined in PC Isolation, Culture, and Identification and subsequently decellularized leaving PC protein-coated transwells. Briefly, cells were incubated in sterile decellularization buffer (PBS supplemented with $0.5 \%$ Triton X-100 and $0.07 \%$ ammonium hydroxide) for 5 minutes and rinsed thoroughly with PBS. IL8-supplemented media was added to the basal transwell chambers to establish a chemotactic gradient. In summary, the bases of six-well plates (Corning) were coated with agarose to prevent neutrophil adhesion. In the basal chamber of the plate, recombinant human IL-8 (BioLegend) was added to PC complete media $(100 \mathrm{ng} / \mathrm{mL})$. Transwells were transferred to these plates, and control PC media was added to the apical chamber. The freshly isolated human neutrophils were then added to the apical chamber and incubated at $37^{\circ} \mathrm{C}$ and $5 \%$ carbon dioxide for 1 hour or 24 hours, as indicated. To assess the role of MMP-3 for neutrophil chemotaxis, neutrophils were treated with an anti-MMP-3 antibody $(5 \mu \mathrm{mol} / \mathrm{L}$; Santa Cruz Biotechnology) immediately before addition to PC matrix. After the migration period, the transwells were removed and neutrophils that successfully chemotaxed across the protein layer were collected and counted. All experiments represent at least three different neutrophil donors. Data are expressed as fold change relative to control samples at the same time point.

\section{Neutrophil-Mediated Fragmentation of PC Deposited Matrix}

Freshly isolated human neutrophils were added to PC cultures that contained PC-deposited protein ( 3 million neutrophils per well in PBS-glucose) in the presence or absence of an anti-MMP-3 antibody (5 $\mu \mathrm{mol} / \mathrm{L}$; Santa Cruz Biotechnology) to assess the interplay between neutrophils and PCs in protein remodeling. ${ }^{31}$ Control wells with an equal volume of PBS glucose but no neutrophils or inhibitors were also tested in parallel. Neutrophils were incubated in wells for 24 hours. After neutrophil treatment, PC culture media were collected, and the PC monolayer was suspended in $0.5 \%$ Triton X-100 and $10 \mathrm{mmol} / \mathrm{L}$ ammonia hydroxide in PBS. The suspension was centrifuged to isolate the fibrillar ECM from the soluble cell lysate; the fibrillar ECM was solubilized in 1\% SDS and $25 \mathrm{mmol} / \mathrm{L}$ trisamine hydrochloride $(\mathrm{pH} 8.0)$. The total protein content of the soluble cell lysate and solubilized fibrillar ECM was determined by BCA assay (Fisher Scientific). The cell culture media and solubilized fibrillar ECM were prepared for electrophoresis by adding SDS, glycerol, and dithiothreitol to a final solution concentration of $1.33 \%, 6.6 \%$, and 
$0.07 \mathrm{~mol} / \mathrm{L}$, respectively. The samples were resolved on $4 \%$ to $20 \%$ acrylamide gels (BioRad, Hercules, CA) and transferred to a nitrocellulose membrane (BioRad). The membrane was probed with mouse monoclonal antibodies 7.1 and 2E8 (Develomental Studies Hydridoma Bank, Iowa City, IA) against FN domain $\mathrm{III}_{9-10}{ }^{32}$ and laminin $\gamma 1$, respectively, followed by IR Dye 680LT-labeled donkey anti-mouse polyclonal antibody (LI-COR). The blots were visualized using an Odyssey Infrared Imaging System (LI-COR), and fragments were normalized to total protein (fragments plus intact protein).

Cell culture was additionally tested for the presence of MMPs using a quantitative MMP array (Raybiotech, Norcross, GA). Culture media were used without dilution according to the manufacturer's instructions. The final slides were scanned on a Genepix 4200A microarray scanner (Molecular Devices, Sunnyvale, CA). Analysis was conducted using LI-COR Image Studio Lite. The effect of adding an MMP-3 inhibitor ( $5 \mu \mathrm{mol} / \mathrm{L}$; Santa Cruz Biotechnology) was evaluated by adding the inhibitor directly to an MMP-3 standard.

\section{Gelatin Zymography}

The soluble cell lysate and the PC culture media were analyzed for active and inactive MMP-2 and MMP-9 as previously described. ${ }^{33}$ The soluble cell lysate and culture media were made to $50 \mathrm{mmol} / \mathrm{L}$ Tris hydrochloride, $8 \%$ glycercol, $1.6 \%$ SDS, and $0.002 \%$ bromophenol blue and resolved on a $10 \%$ acrylamide gel supplemented with $0.1 \%$ gelatin. The MMPs were activated by incubating the gels in $200 \mathrm{mmol} / \mathrm{L}$ sodium chloride, $5 \mathrm{mmol} / \mathrm{L}$ calcium chloride, and $50 \mathrm{mmol} / \mathrm{L}$ Tris hydrochloride $\left(\mathrm{pH} \mathrm{7.8)}\right.$ at $37^{\circ} \mathrm{C}$. The gels were then stained with Coomassie Brilliant Blue and scanned with the Odyssey Infrared Imaging System (LI-COR). MMP activity was assessed by quantifying reduced gelatin content within the gel.

\section{Computational and Statistical Analysis}

Raw data were normalized using unit variance scaling and mean-centered processing before multivariate statistical analysis. Principal component analysis (PCA) and partial least squares regression (PLSR) were completed using SIMCA software version 14.1 (MKS Data Solutions, Malmo, Sweden) as hypothesis-generating methods. All models were constructed using two components. For PLSR models, variable importance in projection (VIP) rankings were calculated by summing the squares of the PLSR loading weights, weighted by the amount of sum of squares in each model component, as described elsewhere ${ }^{34}$; VIP error bars represent 95\% CIs. Graphs and statistical tests were generated in GraphPad software version 7.0 (GraphPad Software, Inc., La Jolla, CA). Unless otherwise noted, data are expressed as means \pm SEM. Statistical significance was determined with one-way- or two-way analysis of variances or unpaired $t$-tests as appropriate. $P<0.05$ was considered statistically significant.

\section{Results}

\section{Morphometric Assessment of Microvascular Remodeling in SS}

Dermal biopsy specimens were collected from seven patients previously diagnosed with SS: three male patients and four female patients ranging in age at the time of biopsy from 46 to 78 years (Table 1). Histologic images revealed that the overall hierarchy of the dermal vasculature was maintained across biopsy specimens from controls and patients with SS; the superficial plexus-where PCVs are localized-retained its horizontal alignment parallel to the epidermis. Further evaluation of these histologic sections revealed varying degrees of leukocytosis in the perivascular space, often resulting in a band-like and MPO-positive infiltrate in the superficial reticular dermis of patients with $\mathrm{SS}$ in contrast to control patients (Figure 1A); MPO is a protease secreted by neutrophils and therefore serves as an indicator of neutrophil accumulation and activity. Consistent with recent reports of SS, our findings support the hypothesis that vascular remodeling indeed occurs in patients with SS. ${ }^{8}$ Throughout the dermal biopsy specimens, the degree of vascular remodeling differed among distinct vessels, with highly remodeled vessels collocating near regions of dense neutrophil infiltrates (leukocytic lesions). Each vessel was assigned a RI to denote visible remodeling (Figure 1) after histologic staining for collagens type I and $\mathrm{IV}$, laminin, and fibronectin to assess the overall degree of vascular remodeling for a given patient. Vessels with an RI of 1 had appropriate vascular structure, although leukocyte recruitment in the region was possible (Figure 1B). Vessels scored with an RI of 2 had some degree of vascular remodeling or damage; the presence of thickened venular walls, loose fibrils, or cellular processes extending from the cell or intramural erythrocytes were all indications of a RI equal to 2 (Figure 1C). Lastly, vessels scored with an RI of 3 indicated severe disruption of the vessel wall, disrupted connected circumference of the vessel structure, and the presence of erythrocytes in the perivascular space (Figure 1D). The microvessels of each patient were evaluated, presenting a mean RI ranging from 1.97 to 2.47 and indicating that vascular remodeling was indeed present in

Table 1 Patient Overview

\begin{tabular}{lllll}
\hline Patient no. & Sex & $\begin{array}{l}\text { Age, } \\
\text { years }\end{array}$ & Location & $\begin{array}{l}\text { Remodeling } \\
\text { index, mean } \pm \text { SD }\end{array}$ \\
\hline 1 & F & 46 & Left forearm & $2.47 \pm 0.05$ \\
2 & M & 66 & Left hip & $2.01 \pm 0.05$ \\
3 & F & 61 & Upper back & $2.24 \pm 0.03$ \\
4 & M & 63 & Finger & $2.17 \pm 0.03$ \\
5 & M & 78 & Right upper arm & $2.13 \pm 0.04$ \\
6 & F & 49 & Left shoulder & $2.26 \pm 0.14$ \\
7 & $F$ & 47 & Right upper arm & $1.90 \pm 0.05$ \\
\hline
\end{tabular}

$F$, female; $M$, male. 


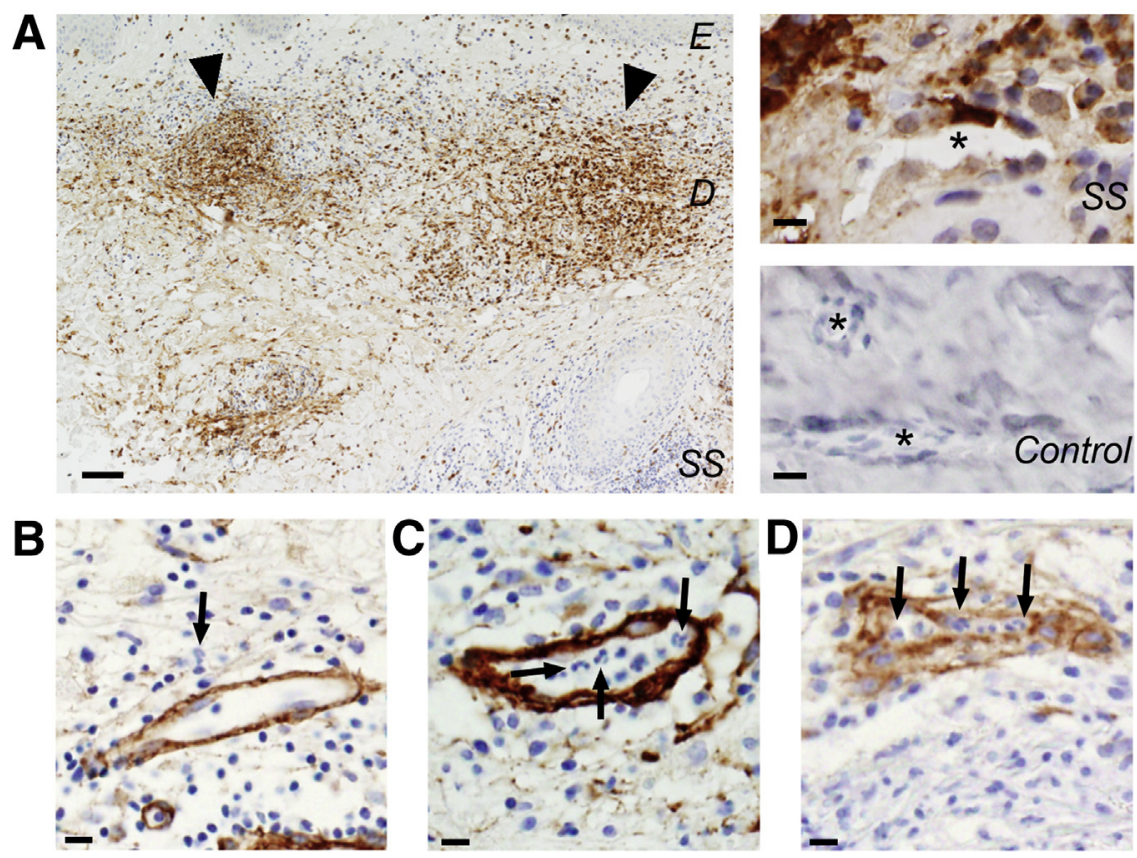

Figure 1 Overview of histologic classifications. A: Compiled overview of one patient section stained for myeloperoxidase (brown) and Hoechst (blue). Arrowheads denote leukocytic bands. Asterisk denotes vessel lumen from patients with Sweet syndrome (SS) and healthy patients stained with MPO (brown) and Hoechst (blue). B-D: Representative images showing vessels scored with a remodeling index of 1 (B), 2 (C), or 3 (D). Sections are stained for collagen IV (brown) and Hoechst (blue). Arrows denote examples of multilobed nuclei indicative of neutrophils. Scale bars: $100 \mu \mathrm{m}(\mathbf{A}) ; 10 \mu \mathrm{m}(\mathbf{B}-\mathbf{D})$. Original magnification: $\times 40(\mathbf{B}-\mathbf{D}) . \mathrm{D}$, dermis; E, epidermis.

SS (Table 1). In comparison, the RIs from healthy volunteers ranged from 1.12 to 1.57 (Supplemental Table S1). Overall, the mean RI of vessels from patients with SS was significantly greater than the mean RI of healthy volunteers $(1.37 \pm 0.04$ versus $2.18 \pm 0.03 ; P<0.0001)$.

\section{PCV BM Composition Is Dynamically Regulated in SS}

Immunohistochemistry for BM proteins was conducted to determine whether compositional changes were associated with the elevated remodeling indices found in the seven dermal biopsy specimens. More than 2500 intensity measurements (measured in arbitrary units [AU]) were taken across PCV segments to quantify changes in collagen types I and IV, laminin, and fibronectin. Furthermore, the measurements were taken in conjunction to intensity measurements for MPO in the region. Taken together, these measurements indicate that there is localized variability in the integrity of PCV walls and the relative amounts of BM proteins and MPO in the vessels and perivascular space. A comparison between healthy volunteers and patients with SS revealed that collagen IV expression was higher in patients with SS, with a mean collagen IV intensity of $148.5 \pm 1.07 \mathrm{AU}$ in patients with SS compared with $106.7 \pm 5.44 \mathrm{AU}$ in healthy volunteers $(P<0.00001)$. Similarly, patients with SS had significantly elevated collagen I within the microvascular wall, with a mean collagen I intensity of $81.99 \pm 1.1$ compared with $60.22 \pm 7.21$ for healthy volunteers $(P<0.05)$.

Changes in PCV BM composition appear to largely coincide with the transition from an RI of 1 to 2 in patients with SS (Figure 2, A, C, E, and G). In moving from an RI of 1 to 2, mean collagen IV expression increased by
$13.8 \% \pm 3.2 \%(P<0.0001)$ (Figure $2 \mathrm{~A})$, mean laminin expression decreased by $14.6 \% \pm 3.9 \%(P<0.01)$ (Figure 2C), and mean collagen I decreased by $14.5 \% \pm 5.3 \%(P<0.05)$ (Figure $2 \mathrm{G})$. Although fibronectin expression decreased by $14 \% \pm 8.2 \%$ in vessels with an RI of 2 compared with those with an RI one of 1 , this change was not statistically significant (Figure 2E). In contrast, similar changes in BM composition were not evident when comparing vessels with RIs of 2 and 3.

Given that SS is a neutrophil-mediated disease, we evaluated BM composition with respect to MPO intensity. Corroborating our qualitative observations, quantification of MPO intensity demonstrated that patients with SS had a marked increase in leukocyte number and/or activity. Whereas MPO staining intensity ranged from 0.2 to 197.9 $\mathrm{AU}$ along the potential intensity range of zero (no staining present) to $254 \mathrm{AU}$, the MPO staining in control samples ranged from 0.0 to $58.2 \mathrm{AU}$. Of the four proteins investigated here, collagen IV and laminin had the greatest changes in intensity correlated with MPO expression (Figure 2, B and D). In patients with SS, collagen IV intensity continued to increase with MPO intensity $(r=0.032)$ (Figure 2B). Laminin expression was similarly lower in healthy volunteers compared with patients with SS in low-MPO regions. As MPO intensity increased, laminin intensity decreased in patients with SS $(r=0.077)$ (Figure 2D).

In contrast, fibronectin intensity within the vascular wall was particularly heterogeneous across the tested samples with no clear correlation with MPO intensity $(r=0.003)$ (Figure 2F). In areas of low MPO, the mean fibronectin intensity in healthy volunteers was higher than that of patients with SS: 72.63 versus 59.8 AU. As MPO intensity increased in patients with SS, fibronectin staining was particularly 

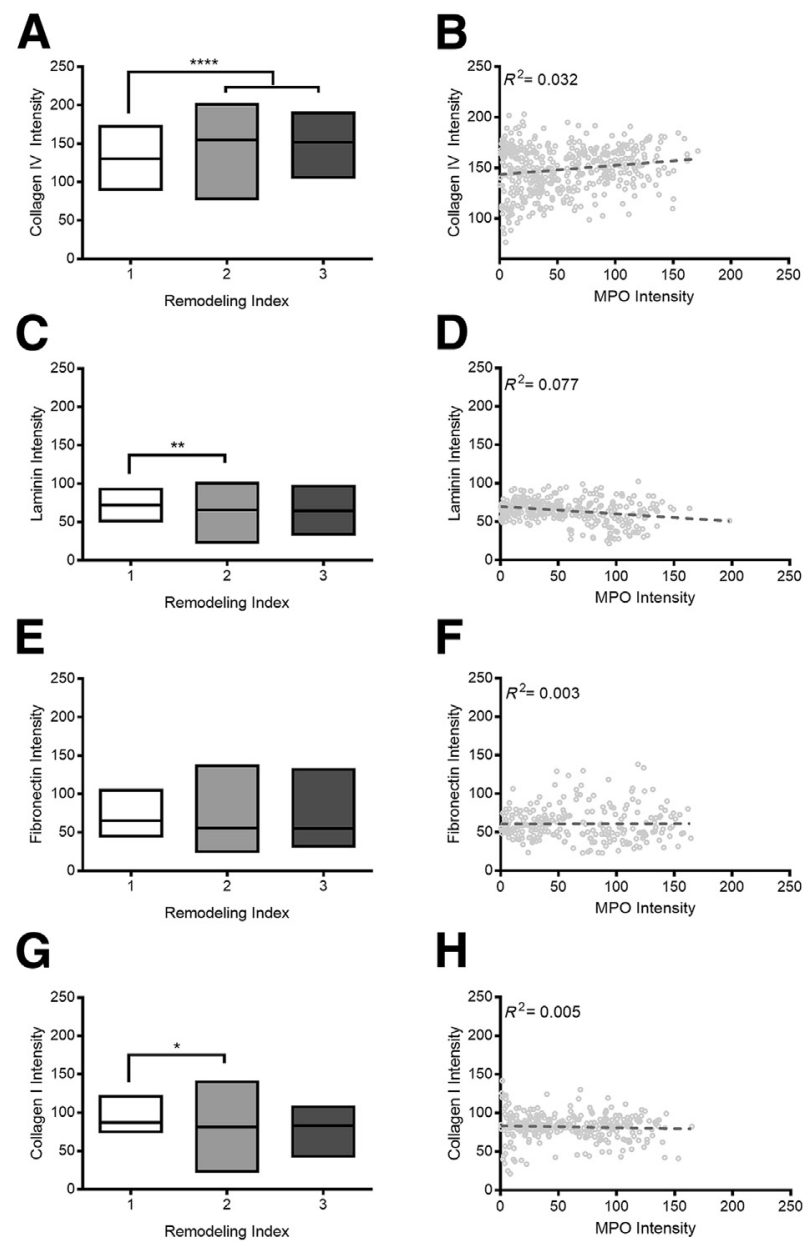

D

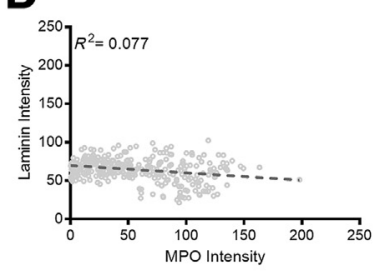

$\mathbf{F}$

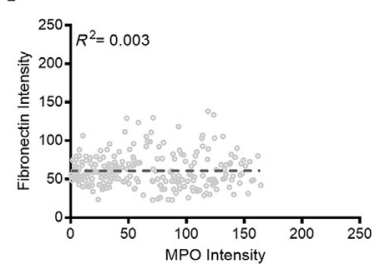

H

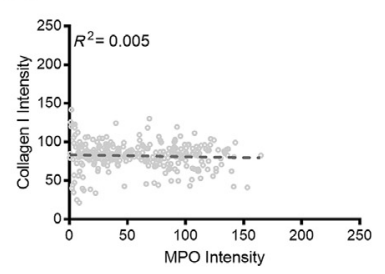

Figure 2 Quantification of histologic analysis for basement membrane proteins. Collagen IV (A), laminin (C), fibronectin (E), and collagen I (G) intensity are shown as a function of the remodeling index. Boxes indicate maximum, median, and minimum values. B, D, F, and $\mathbf{H}$ : Pooled data set from all seven patients with Sweet syndrome showing intensity for basement membrane proteins as a function of myeloperoxidase (MP0); data points shown in light gray. Dashed lines represent linear association between the 2 variables determined by regression analysis, with $R^{2}$ values indicated on each graph. ${ }^{*} P<0.05,{ }^{*} * P<0.01$, and ${ }^{* * * *} P<0.0001$.

varied; the range of fibronectin expression intensities found in high-MPO vessels was four times larger than the range of expression intensities observed in low-MPO tissues. Lastly, collagen type I expression was largely consistent across all MPO values $(r=0.005)$ (Figure $2 \mathrm{H}$ ).

\section{Multivariate Data-Driven Modeling Identifies Fibronectin, MPO, and Collagen IV as Critical Markers of Remodeling}

To investigate the relative importance of the vascular BM proteins for remodeling, we turned to two multivariate datadriven modeling methods, PCA and PLSR. ${ }^{35,36}$ PCA identifies linear combinations of independent variables that vary together in the data set. PLSR identifies linear combinations of variables that most efficiently predict a dependent outcome variable. Using RI as our dependent variable, we conducted PLSR analysis to determine which alterations of the BM were most predictive of RI during the progression of SS (Figure 3). This method was used for the formulation of a hypothesis to focus subsequent experimental design. Fibronectin, MPO, and collagen IV map most closely with patient RI. This graphic association is confirmed with the VIP ranking analysis of these factors (Figure 3). VIP rankings $\geq 1$ indicate significant contributions of a variable (ie, collagen IV, laminin) to the dependent variable (ie, RI). Fibronectin had the highest VIP ranking 1.43 , followed by MPO (1.21) and collagen IV (0.98). Although fibronectin was not significantly different across patients with RI scores ranging from 1 to 3 , linearization of these data through PLSR analysis, which normalizes variations from the mean for all independent variables, scores fibronectin as a potential predictor for patient RI, suggesting that subtle alterations in BM fibronectin could be associated with the severity of vascular dysregulation.

\section{PCs Alter Protein Synthesis in Response to TNF- $\alpha$ and IL-17A Activation}

PCs are largely responsible for synthesis of the healthy PCV $\mathrm{BM}$, and TNF- $\alpha$ and IL-17A are considered the primary cytokines to induce neutrophil recruitment in SS. Therefore, we sought to determine whether activation of human microvascular PCs with TNF- $\alpha$ and IL-17A replicated the microvascular remodeling identified through histologic analysis. We confirmed that isolated PCs retained their expression of essential PC markers. As determined by flow cytometry, cultured PCs stained positively for $\alpha$-SMA (82\%), CD90 (82\%), and NG2 (92\%) (Supplemental Figure S1, A-C), matching the expression of human dermal PC markers (Supplemental Figure S1, D-G). We cultured PCs in the absence of cytokines for 8 days to establish a baseline protein layer. In humans, prior reports

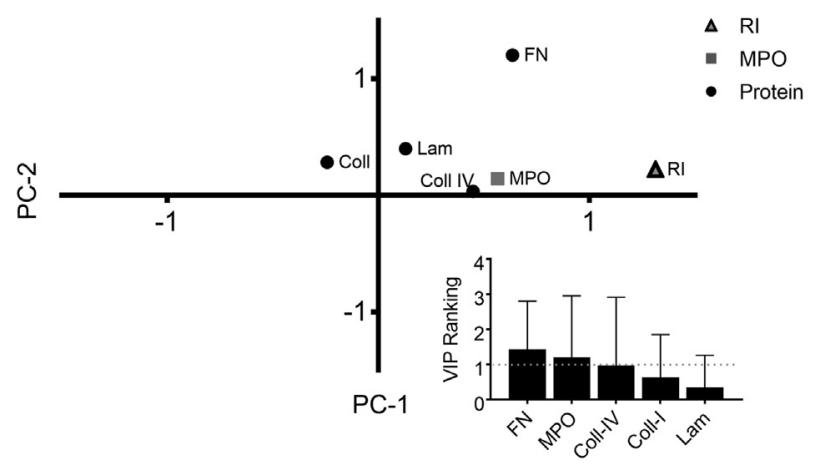

Figure 3 Partial least squares regression (PLSR) of patient histologic data. PLSR analysis of the vascular remodeling indices of the histologic data from the patient with Sweet syndrome. The PLSR model was generated using normalized protein and myeloperoxidase (MP0) intensities as the $x$ variables and remodeling index (RI) as the $y$ variable $\left(R^{2} Y=0.95\right.$; $Q^{2}=0.53$ ). Variable importance in projection (VIP) rankings for the independent variables included in this model (inset). Values above 1 are considered to influence the model. Error bars denote the $95 \% \mathrm{CI}$; dashed line, a VIP value of 1 . Coll, collagen; FN, fibronectin; LAM, laminin. 
indicate that vascular damage was possible after only 3 days of SS lesion onset. ${ }^{8}$ Thus, we then maintained PC cultures under nonactivated and cytokine-activated (TNF- $\alpha$, IL-17A, and TNF- $\alpha$ plus IL-17A conditions) for 1, 3, or 5 days; changes in PC protein were compared with control samples at each time point.

TNF- $\alpha-$ and IL-17A-activation induced alterations in the composition and amount of BM proteins produced by PCs in vitro. Given that prolonged exposure to cytokines has the potential to alter cell proliferation and survival, we first confirmed that our experimental conditions were not affecting cell growth. Through flow cytometry with carboxyfluorescein succinimidyl ester dye, counts of cultured cell nuclei to determine cell density, and BCA assays of cellular protein in culture, we found that there is no significant difference in PC density or proliferation resulting from extended exposure to inflammatory conditions (Supplemental Figure S1, H-J). Having confirmed that potential changes in PC-deposited protein were the result of altered deposition from cultured PCs and not changes in cell proliferation, we proceeded to evaluate PC behavior in culture compared with our histologic samples.

In accordance with our in vivo results, co-activation of PCs with TNF- $\alpha$ and IL-17A resulted in a statistically significant elevation of collagen IV production after 3 and 5 days of activation (Figure 4A). Inflammatory remodeling of collagen IV was most pronounced after 5 days of activation, when TNF- $\alpha$ activation resulted in a $47 \% \pm 13 \%$ $(P<0.001)$ increase over control, IL-17A activation induced a $22 \% \pm 13 \%(P<0.05)$ increase over control, and the dual cytokine activation elevated collagen IV production to $63 \% \pm 13 \%(P<0.01)$ over control. In contrast to the effects on collagen IV, in vitro culture and activation of PCs did not reduce the laminin content as had been seen by histologic analysis of skin biopsy specimens (Figure 4B). Also in contrast to our in vivo observations, our in vitro quantification revealed that fibronectin production by PCs was rapidly and significantly increased in response to cytokine activation (Figure 4C). Within 1 day of activation, IL-17A induced a $44 \% \pm 16 \%$ increase $(P<0.05)$ in fibronectin over control. Evaluation of fibronectin after 3 days of activation, however, demonstrated that IL-17A activation no longer resulted in a significant augmentation of fibronectin levels, but co-activation with TNF- $\alpha$ and IL$17 \mathrm{~A}$ resulted in a $54 \% \pm 17 \%$ increase $(P<0.001)$ in fibronectin over control. The effect of co-activation persisted during the 5 days of activation when the combinatorial activation resulted in a 56\% $\pm 12 \%$ increase $(P<0.001)$ over control. IL-17A activation had an early effect on collagen I accumulation (Figure 4D). After 1 day, IL-17A activation elevated collagen I expression by $21 \% \pm 12 \%(P<0.05)$ compared with control. This change failed to persist over the time course, and there was no significant change in collagen I expression at later time points. The lack of collagen I response in vitro parallels our histologic analysis of in vivo remodeling.
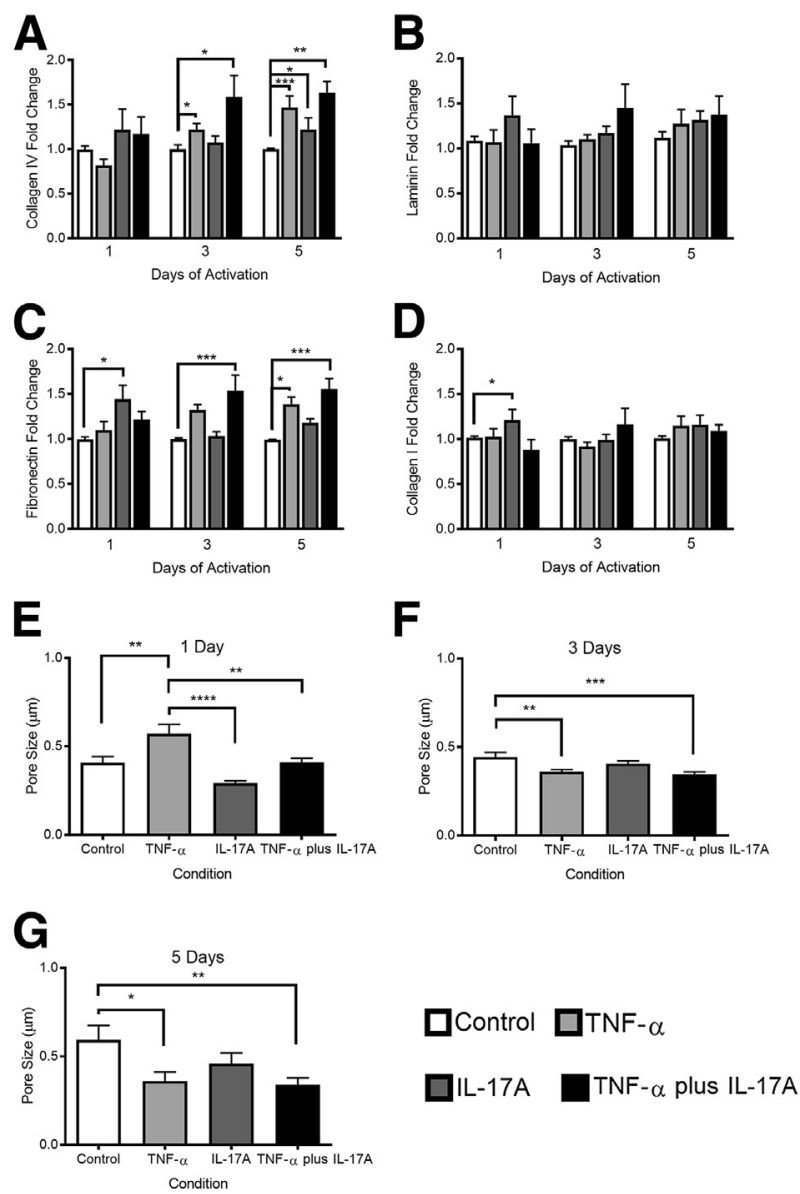

Figure 4 Characterization of pericyte (PC) deposited protein in vitro. A-D: Quantification of PC-deposited collagen IV (A), laminin (B), fibronectin (C), and collagen I (D) in vitro after 1,3 , or 5 days of cytokine activation. E-G: Pore size in $\mathrm{PC}$ protein as determined by eosin staining after 5 days of treatment. Data are expressed as means \pm SEM. $n \geq 6$ (A-D); $n \geq 15(\mathbf{E}-\mathbf{G}) .{ }^{*} P<0.05,{ }^{* *} P<0.01,{ }^{* *} P<0.001$, and $* * * * P<0.0001$

Prior in vitro investigations confirm that compositional changes are associated with changes in the protein architecture, including pore size and protein fibril alignment. ${ }^{37}$ Furthermore, ECM porosity ${ }^{38-40}$ and protein fiber alignment $^{37,41-43}$ are known modulators of cell migration; matrices with smaller pores can promote integrin-dependent migration, ${ }^{38}$ and migration speeds are often more rapid on isotropic substrates compared with anisotropic ones. ${ }^{43}$ In vitro growth of PC matrix is advantageous in that, unlike histologic analyses, both compositional and structural features can be quantified. Thus, we evaluated whether compositional changes were associated with structural remodeling and found that pore size but not fiber alignment is significantly altered in association with changes in PC protein composition (Figure 4 and Supplemental Figure S2). After 1 day of activation, samples treated with TNF- $\alpha$ had the largest mean pore diameter of $0.57 \pm 0.05 \mu \mathrm{m}$ or $39 \%$ larger than the mean control pore diameter $(P<0.01)$. In contrast, IL-17A activation resulted in a $29 \%$ decrease in diameter relative to 

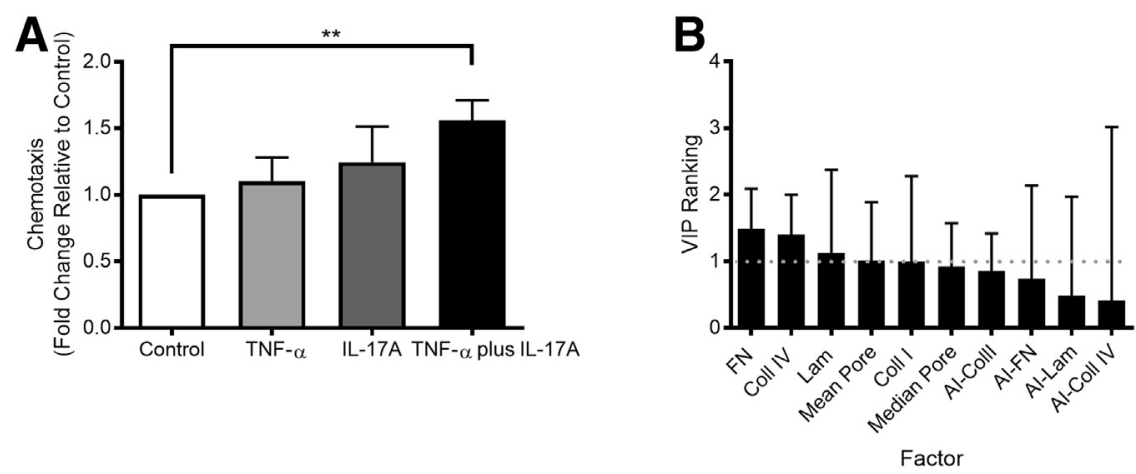

C

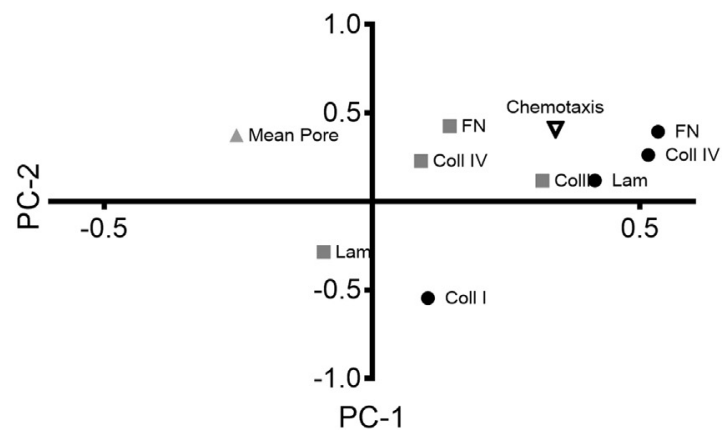

Figure 5 Pericyte (PC) protein remodeling facilitates neutrophil chemotaxis. A: Neutrophil chemotaxis along an IL-8 gradient through decellularized PC protein after 5 days of treatment in culture. B: Variable importance in projection (VIP) rankings from partial least squares analysis. Values above 1 are considered to influence the model. Error bars denote the $95 \%$ CI; dashed line, a VIP value of 1. C: Loading's plot resulting from partial least squares analysis of $\mathrm{PC}$ remodeling data and neutrophil chemotaxis results $\left(R^{2} Y=0.66\right.$; $\left.Q^{2}=0.58\right)$. Data are expressed as means \pm SEM (A and $\mathbf{B}) . n=4(\mathbf{A}) .{ }^{*} P<0.01$. control (not statistically significant), and dual activation of IL-17A and TNF- $\alpha$ did not alter pore diameter (Figure 4E). With time, pore diameter continued to increase in control samples ( 3 days: $0.44 \pm 0.03 \mu \mathrm{m}$; 5 days: $0.60 \pm 0.08 \mu \mathrm{m}$ ), whereas cytokine treatment continually decreased pore size over time. Compared with control samples in the 5-day activation group (Figure $4 \mathrm{G}$ ), TNF- $\alpha$ activation reduced pore size by $39 \%(P<0.05)$, IL-17A decreased pore size by $23 \%$ (not significant), and TNF- $\alpha$ plus IL-17A decreased pore size by $42 \%(P<0.01)$. The mean pore diameter in TNF- $\alpha$ plus IL$17 \mathrm{~A}$-activated PC was $0.34 \pm 0.04 \mu \mathrm{m}$, the smallest mean pore diameter across all time points and conditions. Changes in protein alignment were modest and only observed in the architecture of collagens (Supplemental Figure S2). After 3 days of activation, TNF- $\alpha$ increased collagen IV alignment to $2.13 \pm 0.05$, which was significantly greater than the alignment seen in the control sample $(1.67 \pm 0.05 ; P<0.001)$ and in the TNF- $\alpha$ plus IL-17A condition $(1.74 \pm 0.06$; $P<0.05)$. TNF- $\alpha$ similarly augmented the alignment index for collagen I after 5 days of activation (Supplemental Figure S2D). The alignment index increased to $2.51 \pm 0.07$, which was significantly higher than the control result $(2.18 \pm 0.06 ; P<0.05)$. There were no significant changes in the alignment indices for fibronectin or laminin.

\section{TNF- $\alpha$ Plus IL-17A-Induced BM Remodeling Augments Neutrophil Chemotaxis}

Neutrophil migration is affected by structural and compositional changes in ECM along a chemotactic gradient. ${ }^{37,38}$ Therefore, we investigated the ability of PC protein remodeling to alter neutrophil recruitment along an IL-8 gradient. Using decellularized PC matrices on polycarbonate transwells, chemotaxis assays were conducted using protein generated under all conditions after 1,3 , or 5 days of activation. Significant differences in neutrophil chemotaxis across the nonactivated and activated PC matrices were only seen after 5 days of activation (Figure 5A) (other data not shown). After 5 days of activation, protein remodeling resulting from all three cytokine treatments (TNF- $\alpha$, IL-17A, and TNF- $\alpha$ plus IL-17A) augmented neutrophil chemotaxis compared with control, but only the co-activation of PC with TNF- $\alpha$ plus IL-17A was sufficient to induce a statistically significant increase of $56 \% \pm 14.9 \%$ compared with control samples $(P<0.01)$ (Figure $5 \mathrm{~A})$.

Thus far, we have demonstrated that the prolonged exposure to TNF- $\alpha$ and IL-17A induces PCs to alter the architecture and composition of their BM. To elucidate which of these features are most predicative of the altered neutrophil chemotaxis, we once again used multivariate data-driven modeling. We first conducted PCA using the PC-deposited protein composition, structure, and transmigration data (Supplemental Figure S3). PCA analysis revealed that the data mapped to three groups: one for protein alignment, one for the pore diameter, and one for the composition of PC protein. This served as an internal control to validate that data are mapping as expected. Interestingly, transmigration mapped closely to protein composition, demonstrating that the two are highly correlated.

We proceeded to perform PLSR analysis, using protein composition and structure as our independent variables and chemotaxis (5-day time point values) as our dependent 
variable (Figure 5, B and C). Compositional changes in PC protein are the most influential in predicting neutrophil chemotaxis in the PLSR model, and of the four BM proteins, fibronectin has the highest VIP ranking (1.49). Collagen IV content had the second highest VIP ranking (1.40), followed by laminin content (1.12) and the mean pore diameter (1.01), highlighting their importance in the model. The plot showing how the independent variables map to chemotaxis along PC1 and PC2 reveals that chemotaxis co-varies with compositional cues (fibronectin, collagen IV, and laminin concentration) and structural cues (fibronectin, collagen IV, and collagen I alignment), suggesting that all these factors contribute to the efficiency of neutrophil chemotaxis. The negative PC1 coordinate of mean pore diameter suggests that pore size affects the ability of neutrophils to migrate, but changes in pore size will have the opposite effect on neutrophil chemotaxis compared with changes in protein composition and alignment, which are positive on $\mathrm{PC} 1$.

\section{Synergistic PC Activation and Neutrophil Infiltration Induce Active MMP Expression and Proteolysis}

In vivo, regulation of the $\mathrm{BM}$ is a multifaceted balance of protein synthesis and degradation by cells and their MMPs. ${ }^{44}$ The activation of latent MMPs adds another layer of complexity to the comprehensive regulatory mechanisms that modulate ECM structure and composition. We, therefore, first investigated whether PC expression of MMPs is regulated by cytokine activation. RNA sequencing of PCs activated for 12 hours with TNF- $\alpha$, IL-17A, or TNF- $\alpha$ plus IL-17A revealed that there are significant changes in MMP gene expression $\left({ }^{*} P<0.05\right.$ compared with control) (Supplemental Figure S4, A-D); these changes were validated via gelatin zymography (Supplemental Figure S4E). PCs co-activated with TNF- $\alpha$ and IL-17A had especially robust up-regulation of MMPs associated with fibronectin degradation (Supplemental Figure S4C). RNA sequence analysis reveals that MMP-3 synthesis by PCs undergoes the largest increase after activation with TNF- $\alpha$ and IL-17A relative to control (a 22.2-fold change). MMP-3 is implicated in the proteolysis of fibronectin and laminin, the two proteins for which our in vitro analysis did not fully parallel our histologic findings. ${ }^{31,45}$

Neutrophils are another potential source of proteolytic MMPs in SS and other NDs mediated by TNF- $\alpha$ and IL17A. Neutrophils play two important roles: activating latent MMPs secreted by other cells ${ }^{46,47}$ and secreting MMPs to advance the proteolytic environment. ${ }^{48}$ To gain a more complete understanding of BM remodeling, we added freshly isolated human neutrophils to PCs embedded in their own deposited protein (after 5 days of activation) for 24 hours. After 24 hours, the culture media that contained MMPs from PCs and neutrophils were tested for the presence of active MMP-2 and MMP-9 via gelatin zymography. The presence of neutrophils significantly increased the level of active MMP-9 in all conditions (Figure 6, A and B). The fold change in active MMP-9 after the addition of neutrophils relative to the baseline levels of MMP-9 without neutrophils was determined (Figure 6B); the increase in MMP-9 associated with the TNF- $\alpha$ plus IL-17A dual activation was significantly higher than all other conditions, with a mean fold change of $36.6 \pm 4.5$. There was no significant change in active MMP-2 across conditions, which was expected given the lack of response in the RNA sequencing data.

Given the substantial increase in MMP-3 that was determined via RNA sequencing, we investigated the role of MMP-3 in regulating NDs and SS. We first confirmed the presence of MMP-3 in vivo and in vitro (Figure 6, C and D). MMP-3 staining of human skin biopsy specimens revealed that MMP-3 is present within the PCV wall and in localized patches near perivascular neutrophils, implicating an important role for MMP-3 in SS (Figure 6C). Enzymelinked immunosorbent assay (ELISA) results confirm that in the absence of TNF- $\alpha$ plus IL-17A or neutrophils, MMP-3 levels remain low in PC cultures $(6.2 \mathrm{ng} / \mathrm{mL})$. In contrast, in media collected from TNF- $\alpha$ plus IL$17 \mathrm{~A}$-activated PCs, the level of MMP-3 was elevated to $28.22 \mathrm{ng} / \mathrm{mL}$. The inclusion of neutrophils augmented the baseline production of MMP-3 from control and activated PC cultures, but the concentration of MMP-3 in TNF- $\alpha$ plus IL-17A-activated PCs with neutrophils was significantly elevated (43.99 versus $8.34 \mathrm{ng} / \mathrm{mL} ; P<0.01$ as determined via $t$-test). The inclusion of an MMP-3 inhibitor with neutrophils decreased the levels of MMP-3 within the culture system such that MMP-3 concentrations in control and TNF- $\alpha$ plus IL-17A cultures were statistically equivalent (Figure 6D). The addition of an MMP-3 inhibitor directly to the ELISA did not interfere with measured concentrations of a control with known concentration (Supplemental Figure S4F).

To determine whether elevated MMP levels were sufficient to induce $\mathrm{BM}$ proteolysis and explain the disparity between in vivo and in vitro measurements of fibronectin and laminin, we evaluated the fragmentation of fibrillar fibronectin in culture after 24-hour exposure to neutrophils with or without the addition of an MMP-3 inhibitor. The ratio of fragmented to intact fibronectin and laminin was quantified via Western blot (Figure 6, E and F). There were no significant effects of neutrophil density and no differences observed between the amount of protein fragmentation for fibronectin or laminin with neutrophil densities of 1.5 and 4.5 million (Supplemental Figure S4, G and H). After co-activation with TNF- $\alpha$ plus IL-17A, PC matrix incubated with neutrophils for 24 hours contained significantly more fragmented fibronectin $(0.41 \pm 0.02)$ compared with control $(0.3 \pm 0.03 ; P<0.05)$, when standardized to the total protein in the sample (Figure 6E). The resulting fibronectin fragments had molecular weights of 190 and $88 \mathrm{kDa}$. The $190-\mathrm{kDa}$ fragment, which typically considered a combination of the 150- and 40-kDa fragments can be 

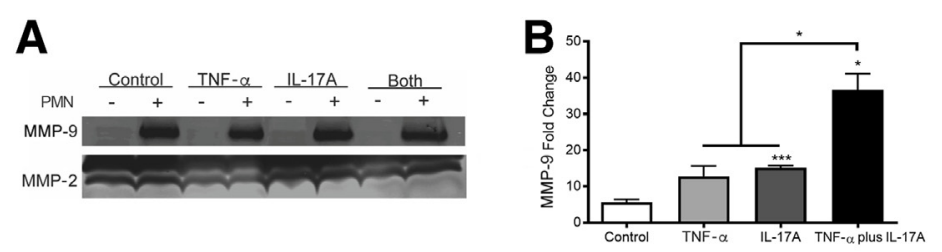

C

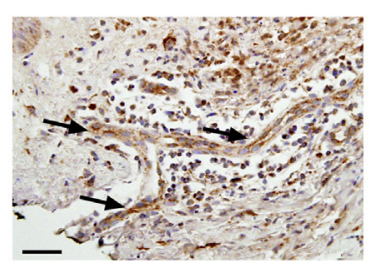

D
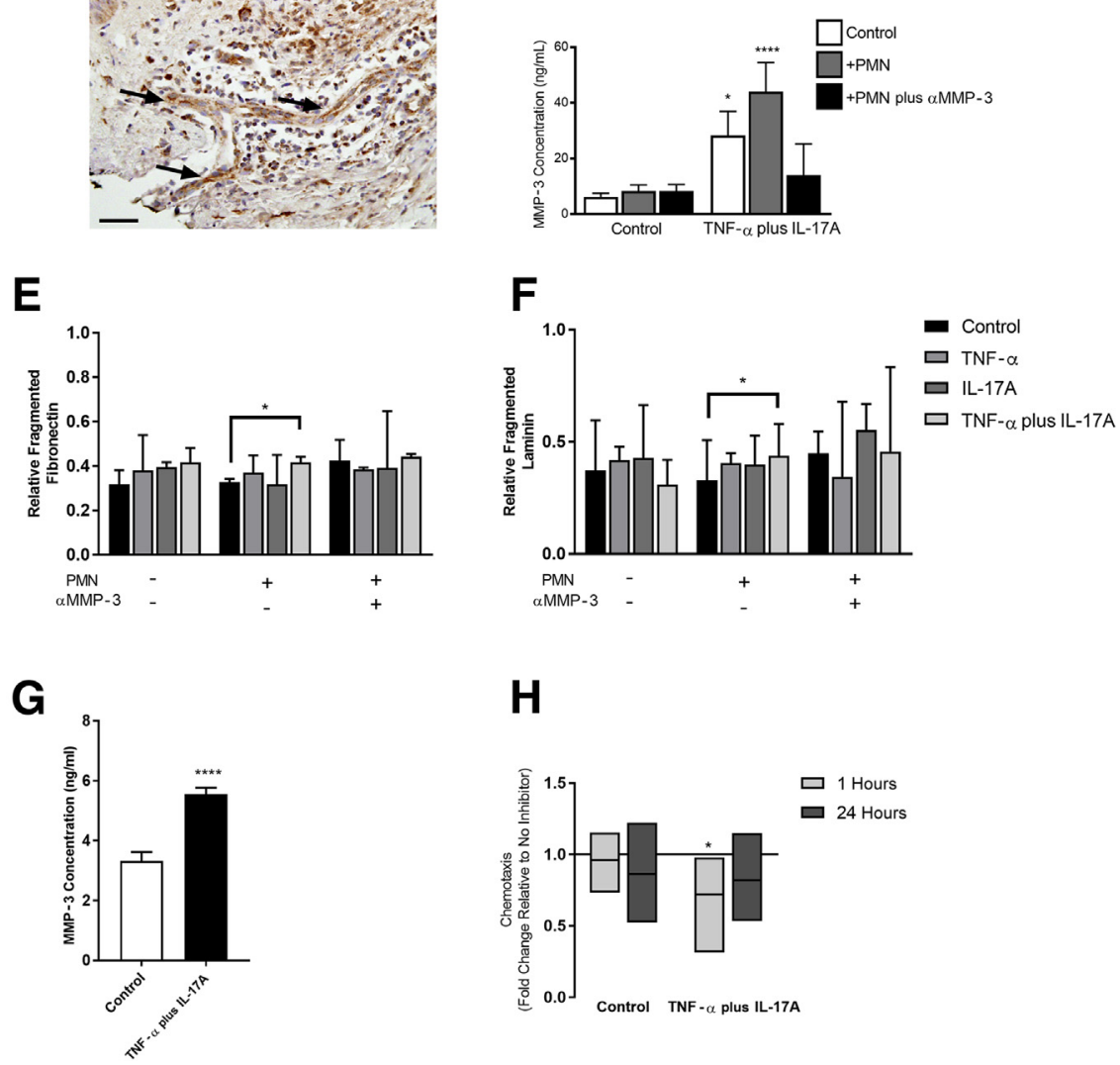

H

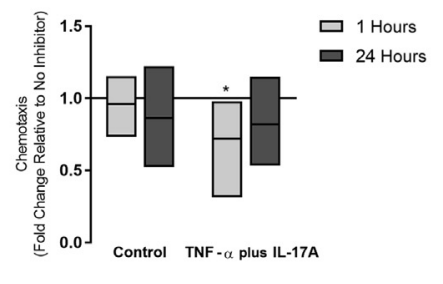

Figure 6 Neutrophils and pericytes (PCs) mediate basement membrane proteolysis. A: Gelatin zymography results for active matrix metalloproteinase (MMP)-9 and MMP-2 in PC culture media after 5 days of activation and 24 hours of incubation with $(+)$ or without $(-)$ neutrophils. B: Active MMP-9 in cell culture media with neutrophils (24 hours); data are represented as fold change over the baseline level found in each PC protein condition without neutrophils. C: Representative histologic image of MMP-3 staining in human Sweet syndrome; Arrow indicates postcapillary venule. D: MMP-3 expression in $\mathrm{PC}$ cell culture media after 5 days of activation and 24 hours of incubation with or without neutrophils, as determined by enzymelinked immunosorbent assay (ELISA) [one-way analysis of variance relative to control with no polymorphonuclear leukocytes (PMN)]. The ratio of fragmented to intact fibrillar fibronectin (E) and fibrillar laminin (F) in PC basement membrane after 5 days of activation and 24 hours of incubation with or without neutrophils. Data were standardized to total protein with a bicinchoninic acid assay. Bars represent median with interquartile range. G: MMP-3 expression of neutrophils in contact with decellularized PC media for 1 hour, as determined by ELISA. H: Neutrophil chemotaxis along an IL-8 gradient through decellularized PC protein after 5 days of activation in culture; neutrophils were treated with an anti-MMP-3 inhibitor, and chemotaxis is shown relative to controls without inhibitor. Bars represent maximum, mean, and minimum. H: MMP-3 expression of neutrophils in contact with decellularized PC media for 1 hour, as determined by ELISA. The solid line indicates no change compared with the same condition without an MMP-3 inhibitor. Data are expressed as means \pm SEM (B, D, and G). Bars represent medians with interquartile ranges ( $\mathbf{E}$ and F). Bars represent means \pm minimum/maximum (H). $n=3(\mathbf{B}) ; n \geq 2$ (D, each run in quadruplicate); $n \geq 3$ ( $\mathbf{E}$ and $\mathbf{F}) ; n=3$ (G, each run in quadruplicate); $n \geq 3$ (H). ${ }^{*} P<0.05,{ }^{*} * P<0.001$, and $* * * P<0.0001$. Scale bar $=100 \mu \mathrm{m}(\mathbf{C})$. produced by MMP-3, -13 , or -14 , whereas the approximately $88-\mathrm{kDa}$ fragment is only associated with MMP-3. ${ }^{49}$ Similarly, evaluation of laminin fragmentation after incubation with neutrophils revealed that the fraction of fragmented laminin was significantly greater in the TNF- $\alpha$ plus IL-17A condition compared with control (Figure 6F). Neither TNF- $\alpha$ nor IL-17A alone was sufficient to induce a statistically significant change in the amount of fragmented fibronectin or laminin. We also evaluated the ratio of fragmented to intact fibronectin and laminin to determine whether inhibiting the action of MMP-3 was sufficient to restore said ratio to control levels. With respect to fibronectin and laminin fragmentation, the MMP-3 inhibitor was able to eliminate the effect of the neutrophils in the TNF- $\alpha$ plus IL-17A condition.

Lastly, we sought to understand whether MMP-3 is an important mediator of neutrophil chemotaxis in vitro. After 1 hour of incubation, MMP-3 levels were significantly higher in media collected from neutrophils cultured on decellularized PC protein from TNF- $\alpha$ plus IL-17A-activated PC compared with that from control PC $(5.5 \pm 0.2$ and $3.3 \pm 1.0 \mathrm{ng} / \mathrm{mL}$, respectively; $P<0.0001$ ) (Figure 6G). We previously observed that 1-hour neutrophil chemotaxis is significantly increased across decellularized PC protein from TNF- $\alpha$ plus IL-17A-activated PC cultures (Figure 5A). The addition of an MMP-3 inhibitor slightly, but not significantly, reduced neutrophil chemotaxis across control PC matrix after 1 and 24 hours compared with neutrophils not treated with an MMP-3 inhibitor. In contrast, neutrophils treated with an MMP-3 inhibitor demonstrated a significant reduction of approximately $28 \% \pm 11 \%$ in chemotaxis across TNF- $\alpha$ plus IL-17A-activated PC matrix after 1 hour $(P<0.05)$ (Figure $6 \mathrm{H})$. After 24 hours, chemotaxis was reduced by $18 \% \pm 10 \%$. Together, these data imply that synergistic effects of TNF- $\alpha$ plus IL-17A and neutrophils can induce MMP secretion and activation and fragmentation of PC-deposited proteins and that 
changes in PC protein can further regulate neutrophil MMP production. Thus, these data suggest a positive feedback loop among neutrophils, PC, and PC-deposited protein that may be important in the development and sustainability of NDs.

\section{Discussion}

We present in vivo and in vitro data revealing vascular BM remodeling in SS and implicate the interplay between microvascular PCs and neutrophils as the mediators for this change. Histologic analysis of PCVs in skin lesions from seven patients supported the hypothesis that vascular damage and remodeling are indeed present in classic SS. Within the superficial plexus, PCV remodeling was highly localized and associated with dense neutrophil infiltrates. When quantified, patients' RIs spanned from 1.90 to 2.47 compared with an RI range of 1.12 to 1.57 for healthy volunteers. Perturbations in the vascular structure were not limited to the disruption of the vascular wall. Rather, $>2500$ measurements of BM protein and neutrophil MPO intensity in and around these vessels demonstrated that compositional changes were associated with morphologic remodeling of the vessel. The most significant changes in BM composition occurred early in disease progression in the transition from vessels with an RI of 1 to 2. It is of interest that we observed more profound changes in samples from female patients than from males, but the current numbers are too small to conclude that there is a sexual dimorphism in BM remodeling.

The combination of histologic characterization of vascular remodeling and in vitro analysis of PC-mediated $\mathrm{BM}$ remodeling demonstrated that $\mathrm{PC}$ activation with TNF- $\alpha$ and IL-17A significantly shifted the balance between PC-mediated protein synthesis and PC- and neutrophilmediated proteolysis. Multivariate data-driven analysis implicated fibronectin and collagen IV remodeling as the most influential BM proteins mediating neutrophil chemotaxis in our in vitro system and vascular damage in vivo. TNF- $\alpha$ plus IL-17A activation significantly increased collagen IV production in vivo and in vitro. Overall, this results in deviations from the typical structure and composition of the vascular BM. Collagen IV is the most abundant protein in the vascular $\mathrm{BM}^{50}$ and provides a scaffold for laminin and fibronectin. Increasing collagen concentration increases matrix fiber density, resulting in smaller pore sizes $^{37}$; the addition of fibronectin and laminin coats collagen fibers to further reduce spacing between fibers within the matrix. ${ }^{37}$ Cytokine activation of PCs in culture revealed robust increases in collagen IV and fibronectin, and our analysis of the BM structure confirms that pores within the PC-produced matrix decrease in diameter as collagen IV and fibronectin become more abundant. A prior study reports that integrin-dependent neutrophil chemotaxis is facilitated in matrices with abundant small pores. ${ }^{38}$ Evaluating neutrophil chemotaxis through the decellularized PC matrix further corroborates this hypothesis because neutrophil chemotaxis was significantly augmented across TNF$\alpha$ plus IL-17A-activated PC matrix relative to control. Early PC remodeling in SS, therefore, is likely to facilitate the accumulation of neutrophils within the skin.

Neutrophil recruitment near activated PCs further drives PC-mediated remodeling of the vascular wall. RNA sequencing and ELISA data reveal widespread changes in MMP expression after activation of PCs with TNF- $\alpha$ plus IL-17A. This up-regulation is particularly robust with response to MMP-3, which is known to target laminin and fibronectin and to be activated from its latent form by neutrophil elastase. ${ }^{46,47,51}$ Gelatin zymography confirmed that neutrophils also contribute to the proteolytic microenvironment through their independent production of MMPs, including MMP-9, which is significantly increased in PCneutrophil co-cultures compared with PC cultures alone. Furthermore, neutrophils cultured with decellularized matrix from activated PCs were sufficient to induce an increase in MMP-3. The proteolytic association between PCs and neutrophils that likely contributes to vascular remodeling in vivo was confirmed in culture, as demonstrated by the presence of fragmented fibronectin and laminin. Of the conditions examined, co-activation with TNF- $\alpha$ plus IL-17A was the only condition that resulted in a statistically significant increase in the fragmented fibronectin and laminin relative to control. Western blot analysis demonstrated that the size of these fragments was consistent with prior reports of MMP-3-mediated proteolysis. ${ }^{49}$

Inhibition of MMP-3 reduced the neutrophil-associated fragmentation of laminin and fibronectin and significantly reduced neutrophil chemotaxis across PC matrix. These results highlight the important interplay among neutrophils, PCs, and PC-deposited matrix in the progression of vascular remodeling. Whereas $\mathrm{PC}$ activation alone robustly increased fibronectin and failed to induce significant changes in laminin, our in vivo quantification that captures the contributions of PCs and neutrophils reveals that fibronectin expression was heterogeneous and laminin was reduced as a function of neutrophil MPO. In addition to the structural implications of atypical laminin and fibronectin presentation, matrix remodeling disrupts appropriate biological cues for neutrophil motility. Cleavage at the fibronectin $\mathrm{III}_{9-10}$ site results in arginine-glycine-aspartic acid-containing fibronectin fragments, which have previously been found to cue neutrophils of their arrival to a site of injury ${ }^{52}$ and may act as another feed-forward loop for continued injury in the vascular wall. Similarly, it has been demonstrated that neutrophil integrin-independent migration requires the proper balance of laminin- 8 and -10 , the two isoforms that are most prevalent within the PCVs. ${ }^{53}$ Neutrophils preferentially adhere to laminin-10 over laminin-8; thus, a relative increase in laminin-10 could prevent the requisite detachment phase of neutrophil motility that leads to neutrophil accumulation within the vascular wall. ${ }^{53}$ Halting the actions of MMP-3 to reduce fibronectin and laminin fragmentation 
could therefore provide a more targeted therapeutic approach to preventing vascular damage for patients with SS in lieu of systemic steroids that are currently used clinically.

Our in vitro studies enable us to isolate the effects of specifically PC-mediated changes in matrix deposition in response to the common SS cytokines TNF- $\alpha$ and IL-17A. However, one of the limitations of this model is that it lacks the complexity associated with human disease. Although in vitro human models allow for modulation of individual components for a better understanding of each component that contributes to disease progression, they do not always produce data that mimic in vivo observations. The data presented here imply that neutrophil-mediated secretion and/or activation of MMPs may account for the differences between in vitro and in vivo results. In addition, we acknowledge that microvascular endothelial activation, swelling, and dysfunction may also play a role in BM remodeling in patients with SS. ECs, which share a BM with microvascular PC, are capable of secreting BM proteins and regulating $\mathrm{BM}$ compositions ${ }^{54}$ and may also react to pathogenic changes in PC-deposited BM to become more dysregulated. ${ }^{55}$ ECs could be another source for MMP secretion in vivo because activated dermal ECs increase secretion and transcription of MMP-2 and MMP-9. ${ }^{56}$

In conclusion, by coupling histologic analysis of the skin biopsy specimens of patients with SS with in vitro PC culture, we found that PCs actively remodel the PCV BM in a TNF- $\alpha$ plus IL-17A-rich environment. PC activation yields increased BM synthesis that together structurally and biochemically alter the vascular BM to facilitate neutrophil recruitment. The subsequent interactions between PCs and leukocytes create a proteolytic environment, with high concentrations of MMP-3, leading to the fragmentation of vascular fibronectin and laminin. Looking forward, our data suggest that targeting MMP-3 to attenuate BM fragmentation could be an effective and highly targeted method for preserving vascular integrity and preventing $\mathrm{BM}$ proteolysis in NDs.

\section{Acknowledgment}

We thank Linda Fong for generously providing guidance on multivariate statistical analysis.

\section{Supplemental Data}

Supplemental material for this article can be found at http://dx.doi.org/10.1016/j.ajpath.2017.04.008.

\section{References}

1. Maalouf D, Battistella M, Bouaziz J-D: Neutrophilic dermatosis: disease mechanism and treatment. Curr Opin Hematol 2015, 22: $23-29$
2. Speeckaert R, Lambert J, Grine L, Van Gele M, De Schepper S, van Geel N: The many faces of IL-17 in inflammatory skin diseases. Br J Dermatol 2016, 175:892-901

3. Marzano AV, Menicanti C, Crosti C, Trevisan V: Neutrophilic dermatoses and inflammatory bowel diseases. G Ital Dermatol Venereol 2013, 148:185-196

4. Cohen PR: Sweet's syndrome-a comprehensive review of an acute febrile neutrophilic dermatosis. Orphanet J Rare Dis 2007, 2:34

5. Paydas S, Sahin B, Zorludemir S: Sweet's syndrome accompanying leukaemia: seven cases and review of the literature. Leuk Res 2000, 24:83-86

6. Marzano AV, Ishak RS, Saibeni S, Crosti C, Meroni PL, Cugno M: Autoinflammatory skin disorders in inflammatory bowel diseases, pyoderma gangrenosum and sweet's syndrome: a comprehensive review and disease classification criteria. Clin Rev Allergy Immunol 2013, 45:202-210

7. Cohen PR: Skin lesions of sweet syndrome and its dorsal hand variant contain vasculitis. Arch Dermatol 2002, 138:400-403

8. Malone JC, Slone SP, Wills-Frank LA, Fearneyhough PK, Lear SC, Goldsmith LJ, Hood A, Callen JP: Vascular inflammation (vasculitis) in sweet syndrome. Arch Dermatol 2002, 138:345-349

9. Marzano AV, Cugno M, Trevisan V, Fanoni D, Venegoni L, Berti E, Crosti C: Role of inflammatory cells, cytokines and matrix metalloproteinases in neutrophil-mediated skin diseases. Clin Exp Immunol 2010, 162:100-107

10. Marzano AV: Hidradenitis suppurativa, neutrophilic dermatoses and autoinflammation: what's the link? Br J Dermatol 2016, 174:482-483

11. Mayadas TN, Cullere X, Lowell CA: The multifaceted functions of neutrophils. Annu Rev Pathol 2014, 9:181-218

12. Kolaczkowska E, Kubes P: Neutrophil recruitment and function in health and inflammation. Nat Rev Immunol 2013, 13:159-175

13. Lauridsen HM, Pober JS, Gonzalez AL: A composite model of the human postcapillary venule for investigation of microvascular leukocyte recruitment. FASEB J 2014, 28:1166-1180

14. Armulik A, Abramsson A, Betsholtz C: Endothelial/pericyte interactions. Circ Res 2005, 97:512-523

15. Wang S, Voisin M-B, Larbi KY, Dangerfield J, Scheiermann C, Tran M, Maxwell PH, Sorokin L, Nourshargh S: Venular basement membranes contain specific matrix protein low expression regions that act as exit points for emigrating neutrophils. J Exp Med 2006, 203:1519-1532

16. Schmidt EP, Lee WL, Zemans RL, Yamashita C, Downey GP: On, around, and through: neutrophil-endothelial interactions in innate immunity. Physiology (Bethesda) 2011, 26:334-347

17. Smith LA, Aranda-Espinoza H, Haun JB, Hammer D: Interplay between shear stress and adhesion on neutrophil locomotion. Biophys J 2007, 92:632-640

18. Ley K, Laudanna C, Cybulsky MI, Nourshargh S: Getting to the site of inflammation: the leukocyte adhesion cascade updated. Nat Rev Immunol 2007, 7:678-689

19. Nourshargh S, Hordijk PL, Sixt M: Breaching multiple barriers: leukocyte motility through venular walls and the interstitium. Nat Rev Mol Cell Biol 2010, 11:366-378

20. Ayres-Sander CE, Lauridsen H, Maier CL, Sava P, Pober JS, Gonzalez AL: Transendothelial migration enables subsequent transmigration of neutrophils through underlying pericytes. PLoS One 2013, 8:e60025

21. Voisin MB, Nourshargh S: Neutrophil transmigration: emergence of an adhesive cascade within venular walls. J Innate Immun 2013, 5: 336-347

22. Pieper C, Pieloch P, Galla H: Pericytes support neutrophil transmigration via interleukin-8 across a porcine co-culture model of the blood-brain barrier. Brain Res 2013, 1524:1-11

23. Proebstl D, Voisin M, Woodfin A, Whiteford J, Acquisto F, Jones GE, Rowe D, Nourshargh S: Pericytes support neutrophil subendothelial cell crawling and breaching of venular walls in vivo. J Exp Med 2009, 209:1219-1234 
24. Stark K, Eckart A, Haidari S, Tirniceriu A, Lorenz M, von Brühl ML, Gartner F, Khandoga AG, Legate KR, Pless R, Hepper I, Lauber K, Walzog B, Massberg S: Capillary and arteriolar pericytes attract innate leukocytes exiting through venules and "instruct" them with patternrecognition and motility programs. Nat Immunol 2013, 14:41-51

25. Wang S, Cao C, Chen Z, Bankaitis V, Tzima E, Sheibani N, Burridge K: Pericytes regulate vascular basement membrane remodeling and govern neutrophil extravasation during inflammation. PLoS One 2012, 7:e45499

26. Sava P, Cook IO, Mahal RS, Gonzalez AL: Human microvascular pericyte basement membrane remodeling regulates neutrophil recruitment. Microcirculation 2015, 22:54-67

27. Liu R, Lauridsen HM, Amezquita RA, Pierce RW, Jane-Wit D, Fang C, Pellowe AS, Kirkiles NC, Gonzalez AL, Pober JS: Interleukin-17 promotes neutrophil-mediated immunity by activating microvascular pericytes and not endothelium. J Immunol 2016, 197: 2400-2408

28. Schneider CA, Rasband WS, Eliceiri KW: NIH Image to ImageJ: 25 years of image analysis. Nat Methods 2012, 9:671-675

29. Maier CL, Shepherd BR, Yi T, Pober JS: Explant outgrowth, propagation and characterization of human pericytes. Microcirculation 2010, 7:367-380

30. Simon DD, Horgan CO, Humphrey JD: Mechanical restrictions on biological responses by adherent cells within collagen gels. J Mech Behav Biomed Mater 2012, 14:216-226

31. Ma S, Yang D, Li D, Tang B, Sun M, Yang Y: Biochemical and biophysical research communications cardiac extracellular matrix tenascin-C deposition during fibronectin degradation. Biochem Biophys Res Commun 2011, 409:321-327

32. Nagai T, Yamakawa N, Aota S, Yamada SS, Akiyama SK, Olden K, Yamada KM: Monoclonal antibody characterization of two distant sites required for function of the central cell-binding domain of fibronectin in cell adhesion, cell migration, and matrix assembly. J Cell Biol 1991, 114:1295-1305

33. Toth M, Fridman R: Assessment of gelatinases (MMP-2 and MMP-9) by gelatin zymography. Methods Mol Med 2001, 57:163-174

34. Gaudet S, Janes KA, Albeck JG, Pace EA, Lauffenburger DA, Sorger PK: A compendium of signals and responses triggered by prodeath and prosurvival cytokines. Mol Cell Proteomics 2005, 4: $1569-1590$

35. Geladi P, Kowalski BR: Partial least-squares regression: a tutorial. Anal Chim Acta 1986, 186:1-17

36. Janes KA, Yaffe MB: Data-driven modelling of signal-transduction networks. Nat Rev Mol Cell Biol 2006, 7:820-828

37. Kuntz RM, Saltzman WM: Neutrophil motility in extracellular matrix gels: mesh size and adhesion affect speed of migration. Biophys J 1997, 72:1472-1480

38. Lauridsen HM, Walker BJ, Gonzalez AL: Chemically- and mechanicallytunable porated polyethylene glycol gels for leukocyte integrin independent and dependent chemotaxis. Technology 2014, 2:1-11

39. Mickel W, Munster S, Jawerth LM, Vader DA, Weitz DA, Sheppard AP, Mecke K, FAbry B, Schrodr-Turk GE: Robust pore size analysis of filamentous networks from three-dimensional confocal microscopy. Biophys J 2008, 95:6072-6080
40. Tan J, Shen H, Saltzman WM: Micron-scale positioning of features influences the rate of polymorphonuclear leukocyte migration. Biophys J 2001, 81:2569-2579

41. Lammerman T, Germain RN: The multiple faces of leukocyte interstitial migration. Semin Immunopathol 2014, 36:227-251

42. Kim DH, Provenzano PP, Smith CL, Levchenko A: Matrix nanotopography as a regulator of cell function. J Cell Biol 2012, 197: $351-360$

43. Fraley SI, Wu P-H, He L, Feng Y, Krisnamurthy R, Longmore GD, Wirtz D: Three-dimensional matrix fiber alignment modulates cell migration and MT1-MMP utility by spatially and temporally directing protrusions. Sci Rep 2015, 5:14580

44. Parks WC, Wilson CL, López-boado YS, Forest W: Matrix metalloroteinases as modulators of inflammation and innate immunity. Nat Rev Immunol 2004, 4:617-629

45. Bejarano PA, Noelken ME, Sozuki K, Hudson BG, Nagase H: Degradation of basement membranes by human matrix metalloproteinase 3 (stromelysin). Biochem J 1988, 256:413-419

46. Shapiro SD, Goldstein NM, Houghton AM, Kobayashi DK, Kelley D, Belaaouaj A: Neutrophil elastase contributes to cigarette smokeinduced emphysema in mice. Am J Pathol 2003, 163:2329-2335

47. Grinnell F, Zhu M: Fibronectin degradation in chronic wounds depends on the relative levels of elastase, alpha1-proteinase inhibitor, and alpha2-macroglobulin. J Invest Dermatol 1996, 106:335-341

48. Ardi VC, Kupriyanova TA, Deryugina EI, Quigley JP: Human neutrophils uniquely release TIMP-free MMP-9 to provide a potent catalytic stimulator of angiogenesis. Proc Natl Acad Sci U S A 2007, 104:20262-20267

49. Zhang X, Chen CT, Bhargava M, Torzilli PA: A comparative study of fibronectin cleavage by MMP-1, -3, -13, and -14. Cartilage 2012, 3: 267-277

50. Le Bleu VS, MacDonald B, Kalluri R: Structure and function of basement membranes. Exp Biol Med 2007, 232:1121-1129

51. Saunders WB, Bayless KJ, Davis GE: MMP-1 activation by serine proteases and MMP-10 induces human capillary tubular network collapse and regression in 3D collagen matrices. J Cell Sci 2005, 118: 2325-2340

52. Gudewicz PW, Heinel LA, Stanton K, Singleton D, Frewin MB: Interaction of fibronectin (FN) cell binding fragments and interleukin8 (IL-8) in regulating neutrophil chemotaxis. Biochem Biophys Res Commun 1994, 205:706-713

53. Kenne E, Soehnlein O, Genove G, Rotzius P, Eriksson EE, Lindbom L: Immune cell recruitment to inflammatory loci is impaired in mice deficient in basement membrane protein laminin a4. J Leukoc Biol 2010, 88:523-528

54. Stratman AN, Malotte KM, Mahan RD, Davis MJ, Davis GE: Pericyte recruitment during vasculogenic tube assembly stimulates endothelial basement membrane matrix formation. Blood 2009, 114:5091-5101

55. Pellowe AS, Gonazalez AL: Extracellular matrix biomimicry for the creation of investigational and therapeutic devices. Wiley Interdiscip Rev Nanomed Nanobiotechnol 2016, 8:5-22

56. Li A, Dubey S, Varney ML, Dave BJ, Singh RK: IL-8 directly enhanced endothelial cell survival, proliferation, and matrix metalloproteinases production and regulated angiogenesis. J Immunol 2003, 170:3369-3376 